05,11

\title{
Исследование критических свойств модели Изинга на объемно центрированной кубической решетке с учетом взаимодействия следующих за ближайшими соседей
}

\author{
(C) А.К. Муртазаев ${ }^{1,2}$, М.К. Рамазанов ${ }^{1}$ ฯ , Д.Р. Курбанова ${ }^{1}$, М.К. Бадиев ${ }^{1}$, Я.К. Абуев ${ }^{1}$ \\ ${ }^{1}$ Институт физики ДагНЦ РАН, \\ Махачкала, Россия \\ 2 Дагестанский государственный университет, \\ Махачкала, Россия \\ ฯ E-mail: sheikh77@mail.ru
}

(Поступила в Редакцию 10 мая 2016 г.

В окончательной редакции 14 ноября 2016 г.)

Репличным методом Монте-Карло выполнены исследования критического поведения трехмерной антиферромагнитной модели Изинга на объемно центрированной кубической решетке с учетом взаимодействий следующих за ближайшими соседей. Исследования проведены для соотношений величин обменных взаимодействий следующих за ближайшими и ближайших соседей $k=J_{2} / J_{1}$ в диапазоне значений $k \in[0.0,1.0]$ с шагом $\Delta k=0.1$. В рамках теории конечно-размерного скейлинга рассчитаны статические критические индексы теплоемкости $\alpha$, восприимчивости $\gamma$, параметра порядка $\beta$, радиуса корреляции $v$, а также индекс Фишера $\eta$. Показано, что класс универсальности критического поведения этой модели сохраняется в интервале значений $k \in[0.0,0.6]$. Установлено, что в диапазоне $k \in[0.8,1.0]$ наблюдается неуниверсальное критическое поведение.

Исследование выполнено при финансовой поддержке РФФИ в рамках научных проектов № 16-02-00214-а и 16-32-00105-мол-а.

DOI: 10.21883/FTT.2017.06.44480.169

\section{1. Введение}

В последнее время особое внимание уделяется исследованию магнитных состояний, фазовых переходов (ФП) и критических явлений в фрустрированных спиновых системах. Это связано с тем, что указанные системы зачастую проявляют поведение, отличное от поведения соответствующих нефрустрированных систем. Причина такого поведения заключается в сильном вырождении в спиновой подсистеме, эффективном ослаблении связи и, как следствие, в высокой чувствительности к различным возмущающим факторам: дополнительным взаимодействиям, слабым полям, тепловым и квантовым флуктуациям, анизотропии, дефектам и деформациям [1-4].

Результаты исследований многих авторов показывают, что наличие фрустраций приводит к существенному изменению ряда свойств фундаментального характеpa [4-8]. Можно отметить проблемы, связанные с определением характера ФП, с особенностями и факторами, влияющими на формирование классов универсальности магнитного и кирального критического поведения фрустрированных спиновых систем и др. Кроме того, учет антиферромагнитных взаимодействий следующих за ближайшими соседей в классической трехмерной модели Изинга приводит к вырождению основного состояния, появлению различных фаз, ФП и аномалий критических свойств [9].

В настоящей работе нами предпринята попытка на основе метода Монте-Карло (МК) провести исследова- ние критических свойств антиферромагнитной модели Изинга на объемно центрированной кубической решетке с учетом взаимодействий ближайших и следующих за ближайшими соседей.

Теоретические расчеты и численное моделирование методом МК для этой модели были проведены в [10-16]. Авторы работы [10] методом МК провели исследование критического поведения модели Изинга на различных типах решеток, вычислили температуру ФП и рассчитали значения термодинамических параметров в критической области. Теоретические исследования, проведенные в работах $[11,12]$, также свидетельствуют о том, что для модели Изинга на простой кубической решетке и объемно центрированной кубической решетке имеет место ФП второго рода. Аналогичные результаты получены и в [13-15]. Авторы этих работ рассчитали критические индексы для некоторых термодинамических параметров. Согласно результатам работ $[14,15]$, переход из ферромагнитной фазы в парамагнитную фазу является переходом второго рода, а переход из антиферромагнитной фазы в парамагнитную - переходом первого рода. Отсюда следует, что при увеличении взаимодействия вторых ближайших соседей в системе происходит смена ФП второго рода на ФП первого рода.

Интерес к антиферромагнитной модели Изинга на объемно центрированной кубической решетке обусловлен тем, что учет взаимодействия следующих за ближайшими соседей может привести к возникновению фрустраций, что усложняет решение. При изучении фрустри- 
рованных систем до сих пор основное внимание уделялось спиновым системам на квадратной, треугольной и гексагональной решетке [17-25]. Критические свойства фрустрированных систем на объемно центрированной кубической решетке с учетом взаимодействий вторых ближайших соседей практически не исследованы. Кроме того, первые попытки исследования этой модели предпринимались в то время, когда мощности вычислительных машин и используемые алгоритмы метода МК не позволяли рассчитывать критические параметры с необходимой степенью точности.

Исследование этой модели на основе современных методов и идей позволит получить ответ на ряд вопросов, связанных с критическими свойствами фрустрированных спиновых систем.

\section{2. Модель и метод исследования}

Антиферромагнитная модель Изинга на объемно центрированной кубической решетке с учетом взаимодействий ближайших и следующих за ближайшими соседей описывается гамильтонианом

$$
H=J_{1} \sum_{\langle i, j\rangle}\left(S_{i} S_{j}\right)+J_{2} \sum_{\langle i, l\rangle}\left(S_{i} S_{l}\right),
$$

где $S_{i, j, l}= \pm 1$ - изинговский спин. Первый член в формуле (1) учитывает обменное взаимодействие ближайших соседей величиной $J_{1}>0$, а второй - следующих за ближайшими соседями величиной $J_{2}>0$; $k=J_{2} / J_{1}-$ величина соотношения взаимодействий следующих за ближайшими и ближайших соседей. Схематически эта модель представлена на рис. 1.

Исследование ФП фрустрированных спиновых систем традиционными теоретическими, экспериментальными и численными методами сталкивается с рядом труднопреодолимых проблем. Это связано с тем, что для таких моделей характерна проблема многочисленных долин локальных минимумов энергии. Строго и последовательно на основе микроскопических гамильтонианов такие системы могут быть изучены методами МК [16-18,20,22-27], но обычные методы МК плохо справляются с решением этих проблем. В связи с этим в последнее время разработано много новых вариантов алгоритмов метода МК, которые позволяют преодолеть указанные проблемы. Одними из наиболее мощных и эффективных в исследовании ФП и критических явлений в фрустрированных системах оказались репличные алгоритмы метода МК [28,29]. Поэтому в настоящей работе был использован высокоэффективный репличный обменный алгоритм метода МК.

Расчеты проводились для систем с периодическими граничными условиями и линейными размерами $L \times L=N, L=12-90$, где $L$ измеряется в размерах элементарной ячейки. Соотношение обменного взаимодействия следующих за ближайшими и ближайших соседей менялось в интервале $k \in[0.0,1.0]$ с шагом

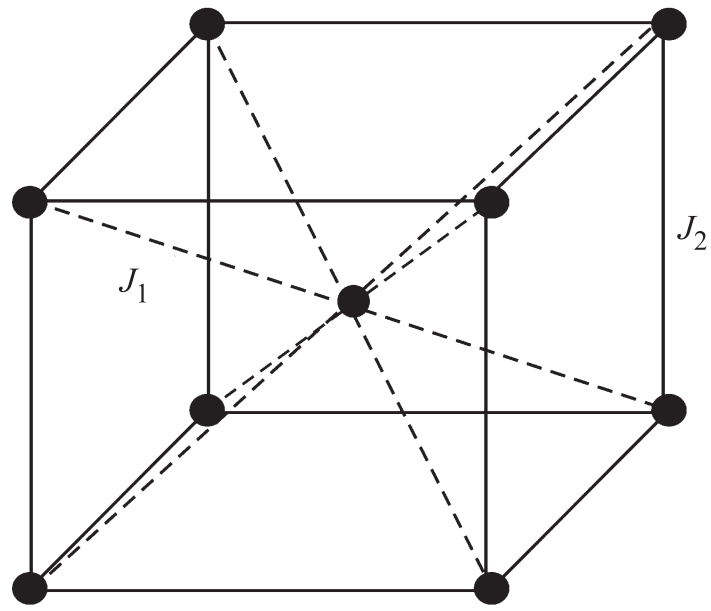

Рис. 1. Схематическое изображение объемно центрированной кубической решетки.

$\Delta k=0.1$. Для вывода системы в состояние термодинамического равновесия отсекался неравновесный участок длиной $\tau_{0}=4 \cdot 10^{5}$ шагов МК на спин, что в несколько раз больше длины неравновесного участка. Усреднение термодинамических параметров проводилось вдоль марковской цепи длиной до $\tau=500 \tau_{0}$ шагов МК на спин.

\section{3. Результаты моделирования}

Для наблюдения за температурным ходом теплоемкости $C$ и восприимчивости $\chi$ использовались выражения $[30,31]$

$$
\begin{gathered}
C=\left(N K^{2}\right)\left(\left\langle U^{2}\right\rangle-\left\langle U^{2}\right\rangle\right), \\
\chi= \begin{cases}(N K)\left(\left\langle M^{2}\right\rangle-\left\langle|M|^{2}\right\rangle\right), & T<T_{N}, \\
(N K)\left\langle M^{2}\right\rangle, & T \geq T_{N},\end{cases}
\end{gathered}
$$

где $K=|J| / k_{\mathrm{B} T, N}$ - число частиц, $T_{N}$ - критическая температура (здесь и далее температура дана в единицах $\left.\left|J_{1}\right| / k_{\mathrm{B}}\right), U-$ внутренняя энергия, $M$ - параметр порядка ( $U$ и $M$ являются нормированными величинами).

Результаты наших исследований показывают, что при $k=0.7$ система имеет некоторые особенности, связанные с поведением термодинамических параметров и типом ФП. Поэтому нами этот случай рассматривается более подробно.

На рис. 2 и 3 представлены характерные зависимости теплоемкости и восприимчивости от температуры для систем с линейными размерами $L=12,24,36$ и 48 при $k=0.7$ (здесь и на всех последующих рисунках статистическая погрешность не превышает размеров символов, использованных для построения зависимостей).

Отметим, что на зависимости теплоемкости $C$ и восприимчивости $\chi$ от температуры для всех систем вблизи критической температуры наблюдаются хорошо выраженные максимумы, которые увеличиваются с ростом числа спинов в системе, причем эти максимумы в 


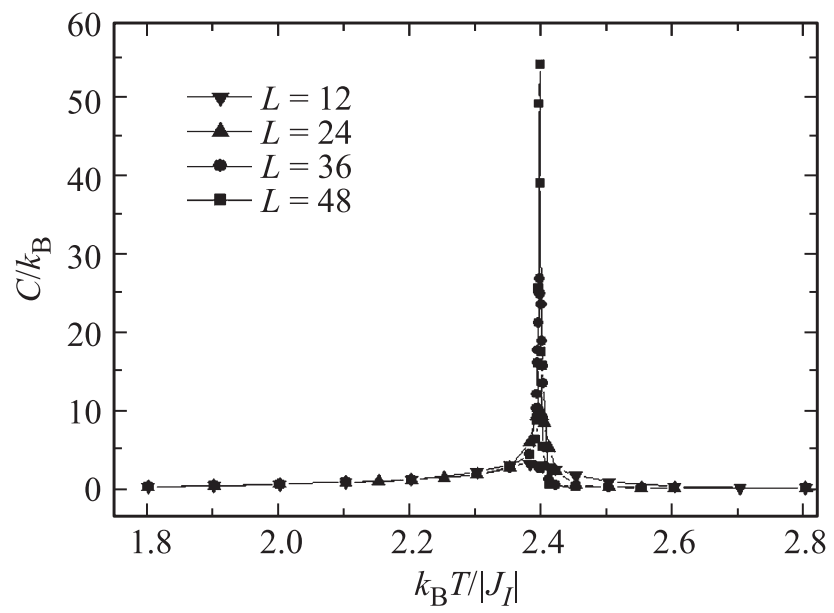

Рис. 2. Зависимость теплоемкости $C / k_{\mathrm{B}}$ от температуры $k_{\mathrm{B}} T /\left|J_{1}\right|$ для $k=0.7$ при различных $L$.

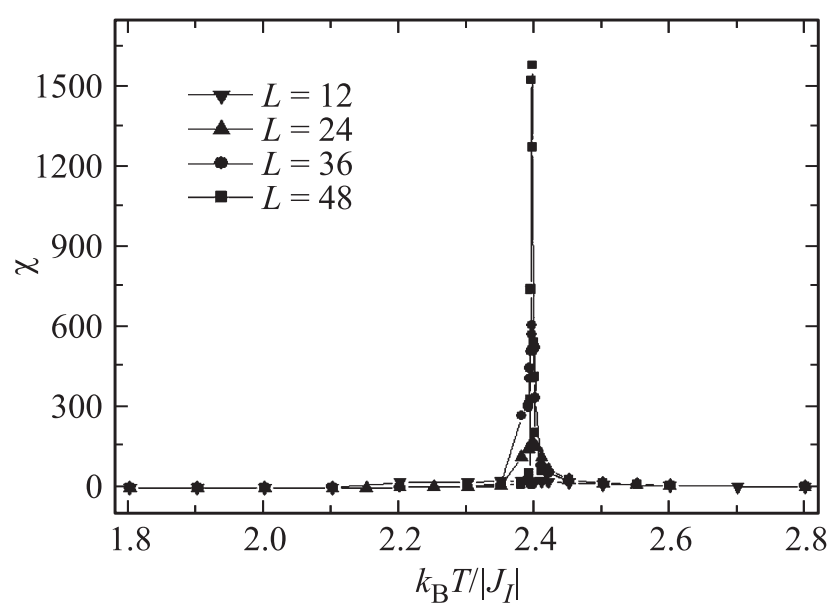

Рис. 3. Зависимость восприимчивости $\chi$ от температуры $k_{\mathrm{B}} T /\left|J_{1}\right|$ для $k=0.7$ при различных $L$.

пределах погрешности приходятся на одну и ту же температуру даже для систем с наименьшим значением $L$. Это свидетельствует, во-первых, о высокой эффективности использованного способа добавления периодических граничных условий, а во-вторых, о достижении насыщения по $N$ для многих исследуемых нами параметров.

Для анализа рода ФП нами использовался гистограммный анализ данных метода МК [32,33]. Этот метод позволяет надежно определить род ФП. Методика определения рода ФП с его помощью подробно описана в $[34,35]$.

Результаты настоящей работы показывают, что переход при $k=0.7$ является ФП второго рода. Это продемонстрировано на рис. 4 и 5. На этих рисунках представлены гистограммы распределения энергии для систем с линейными размерами $L=48,60,72$ и 90. Графики построены вблизи критической температуры. Из рис. 4 и 5 видно, что на зависимости вероятности $P$ от энергии $U$ для систем с линейными размерами $L=48$,
60 и 72 наблюдаются два хорошо выраженных максимума. Наличие двойного максимума на гистограмме распределения энергии является достаточным условием для ФП первого рода. Однако, как видно из рис. 5, для системы с линейными размерами $L=90$ наблюдается один максимум, который свидетельствует в пользу ФП второго рода. Таким образом, при $k=0.7$ в системе с малыми линейными размерами $(L<90)$ наблюдается ФП первого рода. Для всех остальных значений $k$ в зависимости вероятности $P$ от энергии $U$ для систем даже с малыми линейными размерами наблюдается один максимум, который свидетельствует в пользу ФП второго рода. Это продемонстрировано на рис. 6 для случая $k=0.9$.

Более подробно анализ рода ФП для этой модели при всех значениях $k \in[0.0,1.0]$ был проведен нами в работе [16]. Согласно результатам этой работы, во всем рассмотренном интервале значений $k$ наблюдаются ФП второго рода. Случай $k=0.7$ представляет особый интерес и имеет некоторые особенности, которые обсуждались нами выше. Это связано с тем,

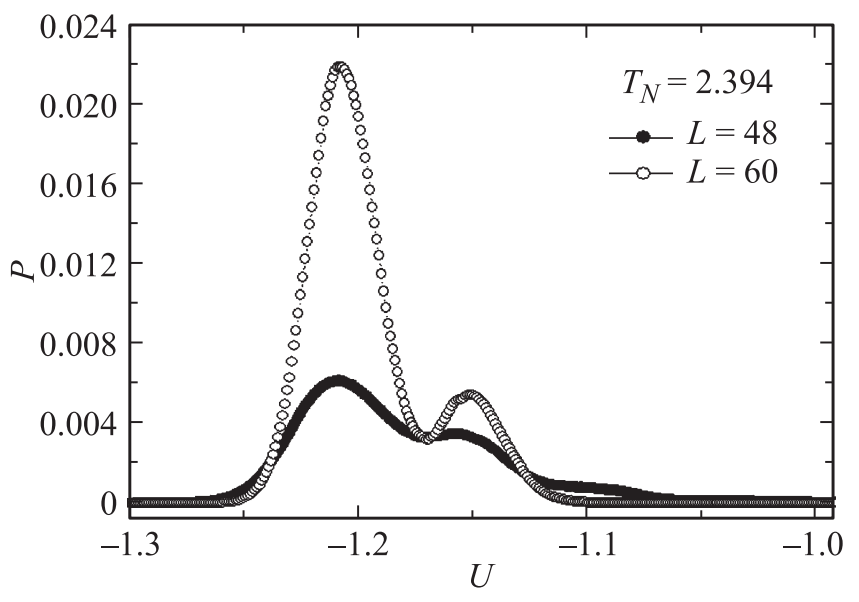

Рис. 4. Гистограмма распределения энергии для $k=0.7$ при $L=48$ и 60 .

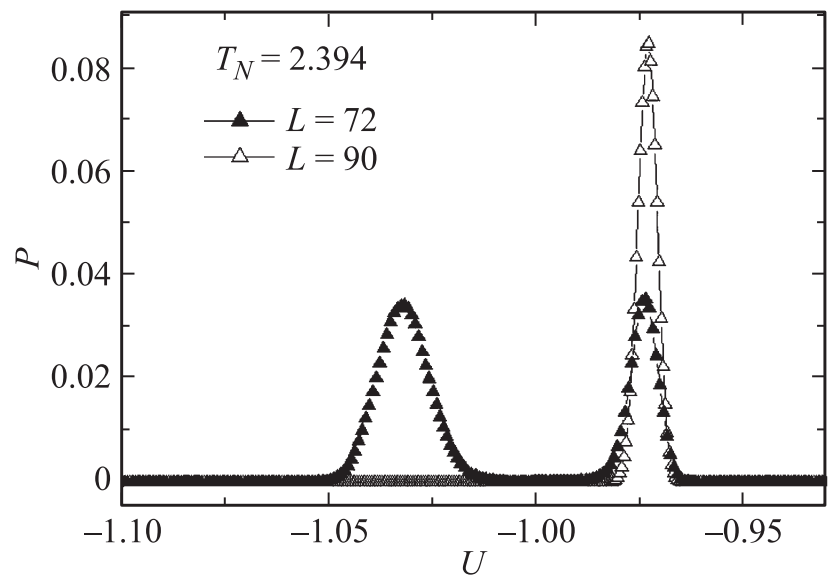

Рис. 5. Гистограмма распределения энергии для $k=0.7$ при $L=72$ и 90 . 


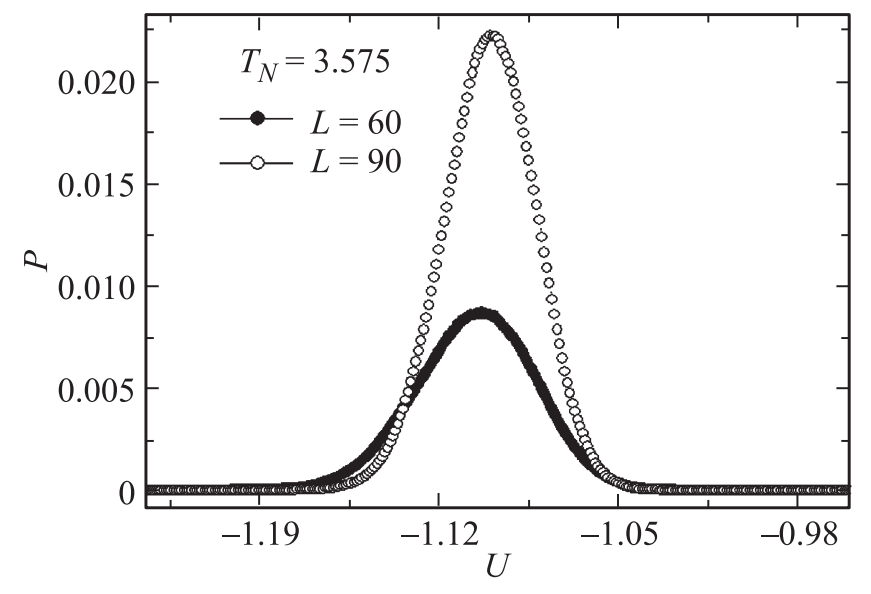

Рис. 6. Гистограмма распределения энергии для $k=0.9$ при $L=60$ и 90.

что вблизи этой точки пересекаются три различные фазы: I - антиферромагнитная, II - парамагнитная, III - антиферромагнитная второго типа [14,15]. Об этом свидетельствует фазовая диаграмма зависимости критической температуры от величины взаимодействия следующих за ближайшими соседей, полученная нами в работе [16].

Для расчета статических критических индексов теплоемкости $\alpha$, восприимчивости $\gamma$, параметра порядка $\beta$, радиуса корреляции $v$ и индекса Фишера $\eta$ применялись соотношения теории конечно-размерного скейлинга $[18,23,31]$.

Из теории конечно-размерного скейлинга следует, что в системе с размерами $L \times L \times L$ при $T=T_{N}$ и достаточно больших $L$ выполняются следующие выражения [23,31]:

$$
\begin{aligned}
M & \sim L^{-\beta / v}, \\
\chi & \sim L^{\gamma / v}, \\
V_{n} & \sim L^{1 / v} g_{V_{n}},
\end{aligned}
$$

где $g_{V_{n}}$ - некоторая постоянная, а в качестве $V_{n}$ могут выступать

$$
V_{n}=\frac{\left\langle M^{n} U\right\rangle}{\left\langle M^{n}\right\rangle}-\langle U\rangle, \quad n=1,2,3 .
$$

Эти выражения были использованы нами для определения $\beta, \gamma$ и $v$.

Для аппроксимации температурной зависимости теплоемкости от $L$ на практике, как правило, используется выражение [20]

$$
C_{\max }(L)=A_{1}-A_{2} L^{\alpha / v},
$$

где $A_{1}$ и $A_{2}-$ некоторые коэффициенты.

На рис. 7 и 8 в двойном логарифмическом масштабе представлены характерные зависимости параметров $V_{n}$ при $n=1,2,3$ от линейных размеров решетки $L$ для $k=0.3$ и 0.9. Как видно из рисунков, все точки на графиках в пределах погрешности хорошо ложатся на прямые. Зависимости на рисунках, построенные в соответствии с методом наименьших квадратов, параллельны друг другу. Углы наклона прямых определяют значения $1 / v$. Вычисленные таким образом значения $v$ использовались для определения критических индексов теплоемкости $\alpha$, восприимчивости $\gamma$ и параметра порядка $\beta$.

На рис. 9-12 в двойном логарифмическом масштабе представлены характерные зависимости магнитного параметра порядка $M$ и восприимчивости $\chi$ от линейных размеров решетки $L$ для $k=0.3$ и 0.9. Все точки в пределах погрешности ложатся на прямые. Углы наклона этих прямых определяют значения $\beta / v$ и $\gamma / v$. По этой схеме были определены значения и для теплоемкости $\alpha / v$. На основе данных по $v$ вычислялись статические критические индексы $\alpha, \beta$ и $\gamma$.

Эта процедура использовалась для расчета критических индексов при значениях $k=1.0,0.9,0.8,0.6$, $0.5,0.4,0.3,0.2,0.1$ и 0.0 . Все значения статических критических индексов, полученные таким образом, представлены в табл. 1. Для случая $k=0.7$ не удалось

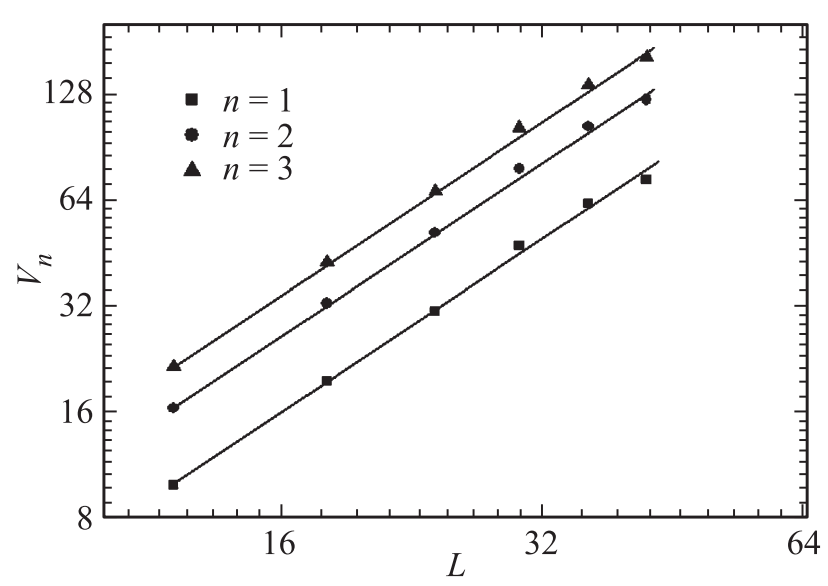

Рис. 7. Зависимость параметра $V_{n}$ от линейных размеров системы $L$ при $T=T_{n}$ для $k=0.3$.

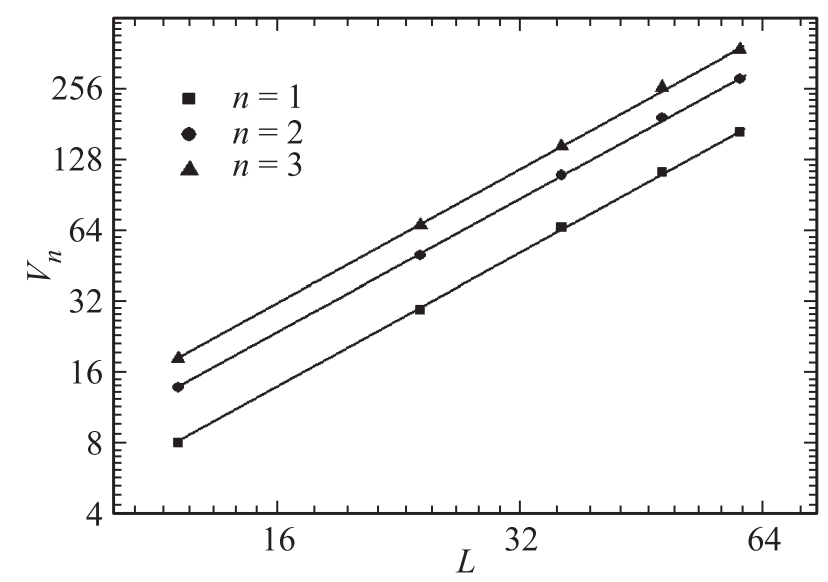

Рис. 8. Зависимость параметра $V_{n}$ от линейных размеров системы $L$ при $T=T_{n}$ для $k=0.9$. 
Таблица 1. Значения критических индексов для трехмерной антиферромагнитной модели Изинга на объемно центрированной кубической решетке

\begin{tabular}{c|c|c|c|c|c|c}
\hline$k$ & $\alpha$ & $\beta$ & $\gamma$ & $v$ & $\eta$ & $\alpha+2 \beta+\gamma=2$ \\
\hline 0.0 & $0.13(2)$ & $0.31(1)$ & $1.23(1)$ & $0.63(1)$ & $0.04(2)$ & 1.98 \\
0.1 & $0.13(2)$ & $0.30(1)$ & $1.24(1)$ & $0.62(1)$ & $0.03(2)$ & 1.97 \\
0.2 & $0.13(2)$ & $0.31(1)$ & $1.23(1)$ & $0.62(1)$ & $0.02(2)$ & 1.98 \\
0.3 & $0.12(2)$ & $0.31(1)$ & $1.25(1)$ & $0.64(1)$ & $0.04(2)$ & 1.99 \\
0.4 & $0.13(2)$ & $0.31(1)$ & $1.22(1)$ & $0.63(1)$ & $0.05(2)$ & 1.97 \\
0.5 & $0.12(2)$ & $0.31(1)$ & $1.24(1)$ & $0.62(1)$ & $0.03(2)$ & 1.98 \\
0.6 & $0.13(2)$ & $0.31(1)$ & $1.22(1)$ & $0.62(1)$ & $0.03(2)$ & 1.97 \\
0.8 & $0.63(2)$ & $0.20(1)$ & $1.01(1)$ & $0.48(1)$ & $-0.07(2)$ & 2.03 \\
1.9 & $0.47(2)$ & $0.22(1)$ & $1.12(1)$ & $0.54(1)$ & $-0.05(2)$ & 2.03 \\
Нефрустрированная & $0.40(2)$ & $0.25(1)$ & $1.14(1)$ & $0.58(1)$ & $-0.01(2)$ & 2.04 \\
модель Изинга [36] & $0.108(9)$ & $0.3265(25)$ & $1.239(4)$ & $0.6305(25)$ & $0.037(3)$ & 2.000
\end{tabular}

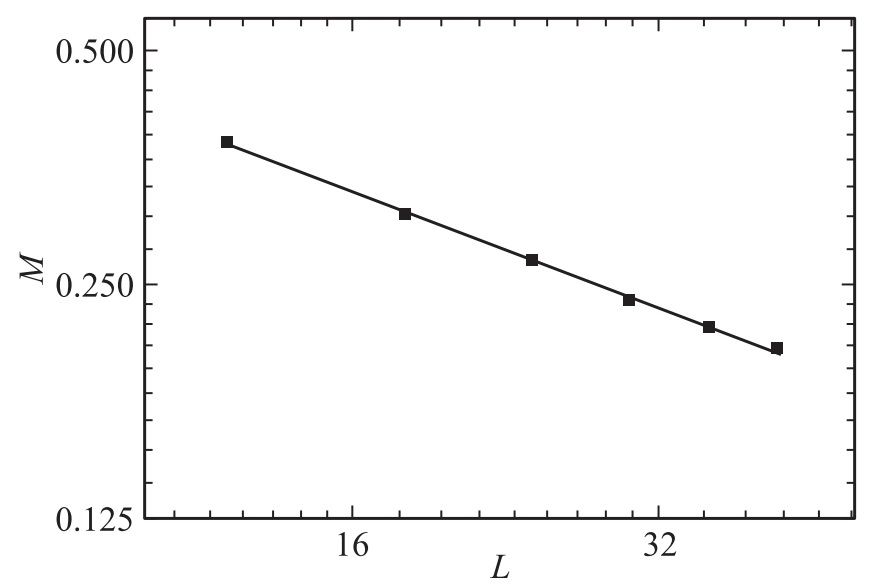

Рис. 9. Зависимость параметра порядка $M$ от линейных размеров системы $L$ при $T=T_{N}$ для $k=0.3$.

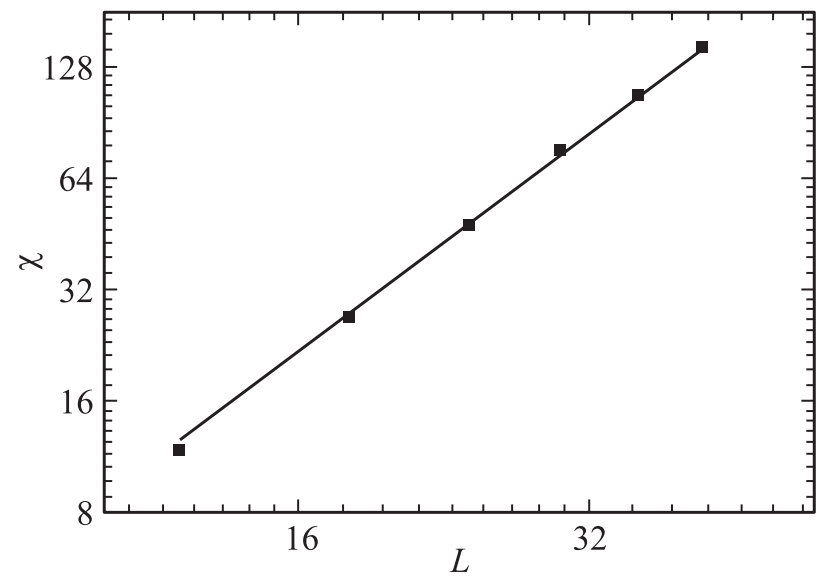

Рис. 10. Зависимость восприимчивости $\chi$ от линейных размеров системы $L$ при $T=T_{N}$ для $k \in=0.3$.

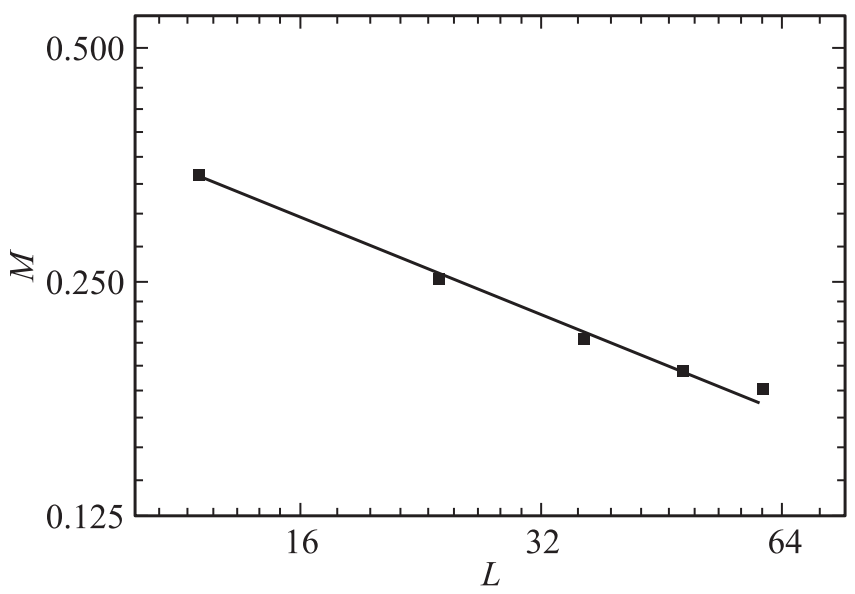

Рис. 11. Зависимость параметра порядка $M$ от линейных размеров системы $L$ при $T=T_{N}$ для $k=0.9$.

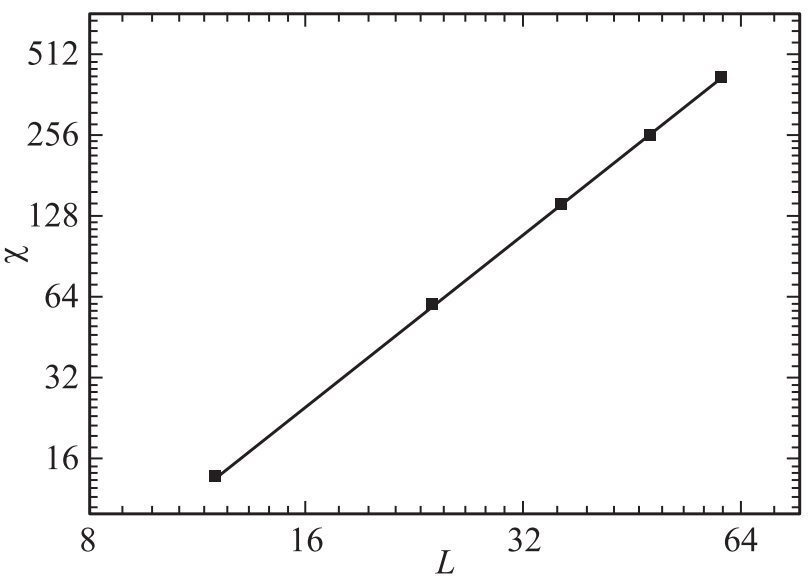

Рис. 12. Зависимость восприимчивости $\chi$ от линейных размеров системы $L$ при $T=T_{N}$ для $k=0.9$. 
рассчитать значения критических индексов. Это связано с тем, что для расчета критических индексов для $k=0.7$ необходимо провести исследования систем с большими линейными размерами $(L \geq 90)$, где происходит $Ф П$ второго рода.

Особо следует отметить процедуру, использованную нами для определения индекса Фишера $\eta$. Используя отношение между восприимчивостью $\chi$ и радиусом корреляции $\xi$ [37]

$$
\chi \propto \xi^{\gamma / \nu}
$$

а также соотношение $\eta=2-\gamma / v$, связывающее индекс $\eta$ и $v$, получим

$$
\ln \left(\chi / \xi^{2}\right)=c-\eta \ln \xi
$$

где $c$ - некоторая константа. Для систем с конечными размерами $\xi=L$. Тогда при $k_{\mathrm{B}} T /\left|J_{1}\right|=k_{\mathrm{B}} T_{N} /\left|J_{1}\right|$ имеем

$$
\ln \left(\chi / L^{2}\right)=c-\eta \ln L \text {. }
$$

На основе выражения (11) было определено значение индекса Фишера $\eta$. Эти данные также представлены в табл. 1 .

Как видно из табл. 1, почти все значения критических индексов, рассчитанные нами в интервале $k \in[0.0,0.6]$, в пределах погрешности совпадают между собой. Это свидетельствует о том, что в этом интервале система проявляет универсальное критическое поведение. В интервале $k \in[0.8,1.0]$ значения критических индексов отличаются от соответствующих значений из интервала $k \in[0.0,0.6]$. Можно предположить, что при увеличении взаимодействия следующих за ближайшими соседей в системе происходит смена класса универсальности критического поведения. Кроме того, в интервале $k \in[0.8,1.0]$ индексы меняются с изменением $k$. Это позволяет нам говорить о том, что в этом интервале наблюдается неуниверсальное критическое поведение. Неуниверсальность критического поведения связана с конкуренцией обменных взаимодействий между ближайшими и следующими соседями. Это следует из решения Бэкстера для точно решаемой восьмивершинной модели [38]. Мы предполагаем, что неуниверсальность критического поведения нашей модели связана с усилением конкуренции обменных взаимодействий между ближайшими и следующими соседями при $k>2 / 3$. Аналогичное поведение предсказывалось также и для трехмерной модели Ашкина-Теллера на кубической решетке [39].

Отметим, что полученные нами значения критических индексов в интервале $k \in[0.0,0.6]$ в пределах погрешности совпадают с соответствующими значениями критических индексов для нефрустрированной трехмерной модели Изинга [36]. Это свидетельствует о том, что исследуемая нами модель принадлежит к тому же классу универсальности критического поведения, что и трехмерная модель Изинга на кубической решетке.

В табл. 2 для сравнения приведены литературные данные для критических индексов, полученные в аналогичной модели. Видно, что наши результаты для интервала $k \in[0.0,0.6]$ находятся в хорошем согласии как
Таблица 2. Значения критических индексов $\gamma$ и $v$ для трехмерной антиферромагнитной модели Изинга на объемно концентрированной кубической решетке (литературные данные)

\begin{tabular}{l|l|l|l}
\hline \multirow{2}{*}{\multicolumn{2}{c|}{ Литературная ссылка }} & \multicolumn{2}{c}{ Критический параметр } \\
\cline { 3 - 4 } \multicolumn{2}{c}{} & \multicolumn{1}{c}{$\gamma$} & \multicolumn{1}{c}{$v$} \\
\hline \multirow{2}{*}{ Теория } & {$[11]$} & $1.23742(20)$ & $0.6308(10)$ \\
& {$[40]$} & $1.2378(6)$ & $0.6311(3)$ \\
& {$[41]$} & $1.2380(50)$ & $0.6305(25)$ \\
\multirow{4}{*}{ Метод МК } & {$[42]$} & $1.2371(4)$ & $0.63002(23)$ \\
& {$[14]$} & $1.25(2)$ & $0.64(2)$ \\
& {$[43]$} & $1.2367(11)$ & $0.6296(7)$ \\
& {$[44]$} & $1.237(2)$ & $0.6301(8)$ \\
& {$[45]$} & $1.2372(17)$ & $0.6303(6)$
\end{tabular}

с теоретическими данными [11,40-42], так и с данными других авторов, полученными методом МК [14,43-45]. Некоторые критические параметры для данной модели при различных значениях $k$ рассчитаны нами впервые.

\section{4. Заключение}

Исследование фазовых переходов и критических свойств в трехмерной антиферромагнитной модели Изинга на объемно центрированной кубической решетке с учетом взаимодействия следующих за ближайшими соседей выполнено с использованием высокоэффективного репличного алгоритма метода Монте-Карло. Рассчитаны значения всех основных статических критических индексов в интервале $k \in[0.0,1.0]$ с шагом $\Delta k=0.1$, кроме случая $k=0.7$. Установлены закономерности изменения критических параметров в рассмотренном интервале $k$. Обнаружено, что в интервале $k \in[0.0,0.6]$ система проявляет универсальное критическое поведение, а в интервале значений $k \in[0.8,1.0]$ наблюдается неуниверсальное критическое поведение.

\section{Список литературы}

[1] С.С. Сосин, Л.А. Прозорова, А.И. Смирнов. УФН 92 (2005).

[2] Вик.С. Доценко. УФН 165, 481 (1995).

[3] С.Е. Коршунов. УФН 176, 233 (2006).

[4] С.В. Малеев. УФН 172, 617 (2002).

[5] M. Tisser, B. Delamotte, D. Mouhanna. Phys. Rev. Lett. 84, 5208 (2000).

[6] P. Calabrese, P. Parruccini, A. Pelissetto, E. Vicari. Phys. Rev. B 70, 174439 (2004).

[7] G. Zumbach. Nucl. Phys. B 413, 771 (1994).

[8] A. Pelissetto, P. Rossi, E. Vicari. Phys. Rev. B 63, 140414(R) (2001).

[9] D.P. Landau, K. Binder. A guide to Monte Carlo simulations in statistical physics. Cambridge University Press, Cambridge (2000). 384 p.

[10] P.H. Lundow, K. Markstrom, A. Rosengren. Phil. Mag. 89, 2042 (2009). 
[11] P. Butera, M. Comi. Phys. Rev. B 65, 144431 (2002).

[12] P. Butera, M. Comi. Phys. Rev. B 72, 014442 (2005).

[13] M. Plischke, J. Oitmaa. Phys. Rev. B 19, 487 (1979).

[14] J.R. Banavar, D. Jasnow, D.P. Landau. Phys. Rev. B 20, 3820 (1979).

[15] M.J. Velgakis, M. Ferer. Phys. Rev. B 27, 401 (1983).

[16] А.К. Муртазаев, М.К. Рамазанов, Ф.А. Касан-Оглы, Д.Р. Курбанова. ЖЭТФ 147, 127 (2015).

[17] H. Kawamura. J. Phys. Soc. Jpn. 61, 1299 (1992).

[18] A. Mailhot, M.L. Plumer, A. Caille. Phys. Rev. B 50, 6854 (1994).

[19] Л.Е. Свистов, А.И. Смирнов, Л.А. Прозорова, О.А. Петренко, А.Я. Шапиро, Л.Н. Демьянц. Письма в ЖЭТФ 80, 231 (2004).

[20] А.К. Муртазаев, М.К. Рамазанов, М.К. Бадиев. ФНТ 37, 1258 (2011).

[21] F.A. Kassan-Ogly, B.N. Filippov, A.K. Murtazaev, M.K. Ramazanov, M.K. Badiev. J. Magn. Magn. Mater. 324, 3418 (2012).

[22] А.К. Муртазаев, М.К. Рамазанов, М.К. Бадиев. ЖЭТФ 142, 338 (2012).

[23] A.K. Murtazaev, M.K. Ramazanov, M.K. Badiev. Physica B 476, 1 (2015).

[24] F.A. Kassan-Ogly, A.K. Murtazaev, A.K. Zhuravlev, M.K. Ramazanov, A.I. Proshkin. J. Magn. Magn. Mater. 384, 247 (2015).

[25] M.K. Ramazanov, A.K. Murtazaev, M.A. Magomedov. Solid State Commun. 233, 35 (2016).

[26] А.К. Муртазаев, М.К. Рамазанов, М.К. Бадиев. ФТТ 52, 1557 (2010).

[27] А.К. Муртазаев, М.К. Рамазанов. ФТТ 53, 1004 (2011).

[28] A. Mitsutake, Y. Sugita, Y. Okamoto. Biopolymers Peptide Sci. 60, 96 (2001).

[29] А.К. Муртазаев, М.К. Рамазанов, Ф.А. Касан-оглы, М.К. Бадиев. ЖЭТФ 144, 1239 (2013).

[30] K. Binder, J.-Sh. Wang. J. Stat. Phys. 55, 87 (1989).

[31] P. Peczak, A.M. Ferrenberg, D.P. Landau. Phys. Rev. B 43,6087 (1991).

[32] F. Wang, D.P. Landau. Phys. Rev. Lett. 86, 2050 (2001).

[33] F. Wang, D.P. Landau. Phys. Rev. E 64, 056101 (2001).

[34] М.К. Рамазанов. Письма в ЖЭТФ 94, 335 (2011).

[35] М.К. Рамазанов, А.К. Муртазаев. Письма в ЖЭТФ 101, 793 (2015).

[36] J.C. Le Guillou, J. Zinn-Justin. Phys. Rev. B 21, 3976 (1980).

[37] Ch. Holm, W. Janke. Phys. Rev. B 48, 936 (1993).

[38] Р. Бэкстер. Точно решаемые модели в статистической механике. Мир, М. (1985). 488 с.

[39] G. Musial, J. Rogiers. Phys. Status. Solidi B 243, 335 (2006).

[40] M.J. George, J.J. Rehr. Phys. Rev. Lett. 53, 2063 (1984).

[41] R. Guida, J. Zinn-Justin. Nucl. Phys. B 489, 626 (1997).

[42] M. Campostrini, A. Pelissetto, P. Ross, E. Vicari. Phys. Rev. E 60, 3526 (1999).

[43] M. Hasenbusch, K. Pinn, S. Vinti. Phys. Rev. B 59, 11471 (1999).

[44] H.W.J. Blote, E. Luijten, J.R. Heringa. J. Phys. A 28, 6289 (1995).

[45] H.W.J. Blote, L.N. Shchur, A.L. Talapov. Int. J. Mod. Phys. C 10, 137 (1999). 